\title{
Application of Short-Range LIDAR in Early Alerting for Low-Level Windshear and Turbulence at Hong Kong International Airport
}

\author{
K. K. Hon, ${ }^{1}$ P. W. Chan, ${ }^{1}$ Y. Y. Chiu, ${ }^{1}$ and Wenbo Tang ${ }^{2}$ \\ ${ }^{1}$ Hong Kong Observatory, 134A Nathan Road, Kowloon, Hong Kong \\ ${ }^{2}$ School of Mathematical \& Statistical Sciences, Arizona State University, Tempe, AZ 85287, USA \\ Correspondence should be addressed to P. W. Chan; pwchan@hko.gov.hk
}

Received 2 August 2014; Revised 6 November 2014; Accepted 11 November 2014; Published 1 December 2014

Academic Editor: Hiroyuki Hashiguchi

Copyright $\odot 2014 \mathrm{~K}$. K. Hon et al. This is an open access article distributed under the Creative Commons Attribution License, which permits unrestricted use, distribution, and reproduction in any medium, provided the original work is properly cited.

Hong Kong Observatory currently uses a series of meteorological instruments, including long-range LIDAR (light detection and ranging) systems, to provide alerting services of low-level windshear and turbulence for Hong Kong International Airport. For some events that are smaller in spatial dimensions and are rapidly changing, such as low altitude windshear and turbulence associated with buildings or man-made structures, it would be necessary to involve meteorological instruments that offer greater spatial resolution. Therefore, the Observatory has set up a short-range LIDAR on the roof of the AsiaWorld-Expo during the summers over the past several years, conducting field research on the feasibility of strengthening early alerting for windshear and turbulence over the north runway's eastern arrival runway (Runway 25RA) and developing an automated early alerting algorithm. This paper takes the pilot reports for Runway 25RA during the 2013 field research as verification samples, using different thresholds for radial wind velocity spatial and temporal changes detected by the short-range LIDAR to calculate the relative operating characteristic (ROC) curve, and analyzes its early alerting performance.

\section{Foreword}

Hong Kong International Airport is surrounded by sea on three sides and with Lantau Island of complex terrain to the south. The Lantau Island is composed of rows of northeastsouthwest mountain ranges, with peaks ranging from 700 to 950 meters and valleys ranging from 350 to 450 meters above mean sea level (see Figure 1(a)). Most windshear and turbulence at Hong Kong International Airport are brought about by strong winds across the Lantau Island to the south of the airport, including the strong winds generated by tropical cyclones and strong monsoons, contributing to $70 \%$ of all of the windshear reported by pilots. Moreover, $20 \%$ of the pilot windshear reports are brought about by sea breeze, and only $10 \%$ are brought about by squall lines, microbursts, and low altitude jets.

As a part of the "Windshear and Turbulence Warning System (WTWS)," Hong Kong Observatory introduced the longrange LIDAR (light detection and ranging) system in 2002 to provide Hong Kong International Airport with windshear early alerting services. Based on the Doppler effect principle, LIDAR uses infrared rays to detect the movement of airborne dust particles and particulates, so as to calculate radial wind velocity data. Research shows that a long-range LIDAR is highly effective [1] in capturing the windshear brought about by the terrain-related airflow disturbances under nonrainy weather conditions.

With the development of Hong Kong International Airport, the number of buildings in the Island, such as office buildings, hotels, and hangers, has also increased. When airflow passes these buildings or man-made structures, it could also bring about low altitude windshear or turbulence to flights taking off or landing on the runways. Meteorological instruments with greater spatial resolution are needed in order to deal with these low altitude windshear and turbulence with smaller spatial dimensions. Therefore, the Observatory have set up a short-range LIDAR on the roof of the AsiaWorld-Expo during the summers over the past several years, conducting field research on the feasibility of strengthening early alerting of windshear over the north runway's 


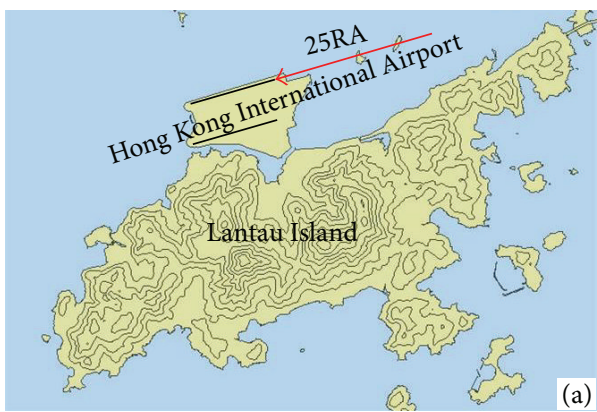

(a)

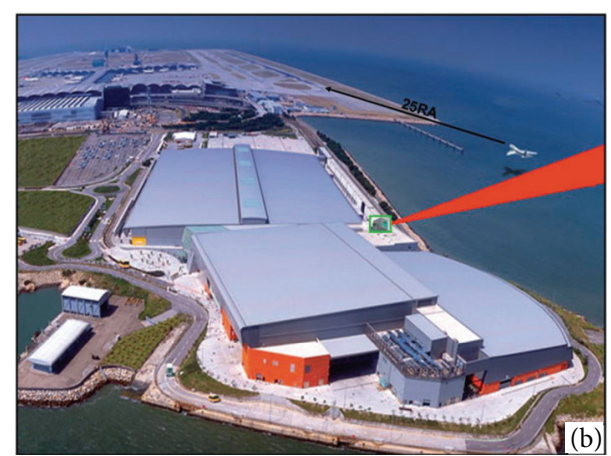

(b)

Figure 1: Hong Kong International Airport and the geographical situation nearby. (a) shows the Lantau Island that is near Hong Kong International Airport (height contours: every $100 \mathrm{~m}$ ). (b) is the diagrammatic sketch that shows the short-range LIDAR and its scan range (red sector) over Runway 25RA of Hong Kong International Airport from the roof of the AsiaWorld-Expo.

eastern arrival runway (Runway 25RA). The 25RA runway corridor is the most used arrival runway corridor in southwesterly flow (southwest monsoon in summer in Hong Kong). The document [2] records the preliminary results of short-range LIDAR experiments conducted by the Observatory during the summer of 2010, including use of three different algorithms, velocity fluctuation, eddy dissipation rate, or EDR, and autocorrelation, to study the effectiveness of shortrange LIDAR in capturing windshear. The results show that the velocity fluctuation is the most effective algorithm. If a short-range LIDAR is used in conjunction with WTWS for early alerting and the pilot reports are considered, the overall performance of early alerting for low altitude windshear can be enhanced.

The Observatory continued field experiments in the summer of 2013, using a set of short-range LIDAR systems with higher radial resolution. This paper will take the data from the period when tropical cyclone Utor hit Hong Kong as an example to analyze the characteristics of building-induced air disturbances detected by short-range LIDAR. Besides that, during the field experiment period, the Observatory used the improved algorithm of short-range LIDAR early alerting for windshear, considering pilot reports from Runway 25RA, to analyze early alerting performance.

\section{Instrument and Data}

From July to August 2013, the Observatory conducted a new field experiment, setting up a short-range LIDAR on the roof of the AsiaWorld-Expo, as it had been done over the past several years, to scan Runway 25RA (Figure 1(b)) and to monitor low altitude windshear and turbulence in the area. The AsiaWorld-Expo is located on the northeast corner of Chek Lap Kok, close to the eastern corridor of the northern runway, about 500 meters far from 25RA at its closest point, so setting up a short-range LIDAR on the AsiaWorld-Expo is relatively effective at detecting the wind field over this runway, particularly between touchdown and 1 nautical mile away from the runway when low-level windshear could be hazardous to the landing aircraft. The elevation angle of this
LIDAR was set to 5 degrees from the horizon, with a scanning azimuthal range from 60 to 300 degrees with respect to the north. A plane position indication (PPI) scan can be made about every 20 seconds, to ensure maximum effectiveness in collecting the latest data on 25RA wind field. The radial distance resolution of this radar is about 30 meters, compared to the field research [2] conducted in 2010, when the radial distance resolution of the short-range LIDAR was 75 meters. The radial distance resolution had increased 1.5 times. Compared to the long-range LIDAR currently used by Hong Kong International Airport (radial resolution of $105 \mathrm{~m}$ ), the radial distance resolution is higher by 2.5 times, more effective for detecting smaller dimension wind field changes related to the buildings. Generally, a short-range LIDAR has an effective measurement distance ranging from 2 to $3 \mathrm{kms}$ under clear sky conditions, with more background noise as the distance increases. Therefore, when processing data, we monitor data quality based on signal-to-noise ratio, or SNR, while inconsistent data (i.e., radial velocity that differs significantly from those velocities in the nearby range gates) would be deleted.

This field experiment collected more than 100,000 scan records, with the experiment period covering the following significant meteorological events: (I) Severe Typhoon Utor drew near to Hong Kong from 12 to 15 August 2013, with Hong Kong Observatory issuing No. 8 Southeast Gale or Storm Signal, (II) due to the influence of Severe Tropical Storm Jebi, the Observatory issued No. 3 Strong Wind Signal on 1 August 2013, and (III) due to the influence of a strong southwesterly, the Observatory issued a Strong Monsoon Signal on 2 August and 23 August 2013.

\section{Monitoring Low Altitude Windshear and Turbulence}

The discussion below elaborates the use of the short-range LIDAR to monitor rapidly evolving airflow disturbances, based on pilot reports, and to analyze the windshear event that occurred over Hong Kong International Airport under the influence of Severe Typhoon Utor. 
Tropical cyclone Utor hit Hong Kong between 12 and 16 August 2013 (see Figure 2). Utor crossed the Philippines and entered the northern part of the South China Sea in the morning of 12 August, with the Observatory issuing No. 1 Standby Signal in that afternoon. Over the next two days, Utor moved towards the northwest, crossing the northern part of the South China Sea and approaching the western coast of Guangdong province. With the gradual strengthening of winds in Hong Kong, the Observatory issued No. 3 Strong Wind Signal and No. 8 Southeast Gale or Storm Signal. Utor made landfall in the afternoon of 14 August around the Yangiiang, Guangdong, area and moved inland. But due to slow movement and associated rain belt continuing to impact the Pearl River Estuary region, as of the morning of 15 August, there were still southerly gales over the southern part of Hong Kong and the western sea region (see Figure 3). At this time, low-level windshear events occurred over the 25RA glide path.

Figure 3(b) shows that, in the morning of 15 August, Hong Kong International Airport and the Lantau Island region were under the influence of fresh to strong southerly winds (green and blue pennants) and on high ground wind speeds can reach strong to gale levels (red pennants). At the same time, influenced by the Lantau Island terrain upstream, wind speed distribution over the airport runway was not so even, especially the eastern (blue-green pennant appeared) side of the north runway. It can be expected that flights landing on this runway (25RA) would feel rapidly changing winds. Fine spatial and temporal changes in the low altitude wind field near the landing glide path 25RA could be monitored with the short-range LIDAR's PPI scans.

Figure 4 shows a sequence of short-range LIDAR radial velocity scans at about $8 \mathrm{am}$ in the morning of 15 August (Hong Kong time), with 40 seconds time difference between images. The white dot in the figures indicates the location of the short-range LIDAR. Runway 25RA location is represented by the white line, while the white circle indicating distance has an increment of 500 meters. The scan image shows that the radial velocity north of the short-range LIDAR was generally positive in value, indicating that the 25RA area was largely experiencing southerly winds, matching the surface wind speed measurements. But, within the short 80 -second period covered by the image sequence, the life cycle of a small dimension airflow disturbance could be seen. Figure 4(a) shows radial wind speeds that are distributed relatively evenly, with relatively low shear in wind speed over runway 25RA. After 40 seconds (Figure 4(b)), a relatively high wind speed region that appears is about 1 nautical mile from the landing site and crosses the glide path of flights. Another 40 seconds later (Figure 4(c)), this disturbance has moved out of the 25RA area, with radial wind speeds over the runway again returning to relatively even levels.

In that morning, the Observatory received a pilot report indicating that, upon descent over Runway 25RA, the flight encountered lifting windshear with strength of 20 knots (or about $10 \mathrm{~m} / \mathrm{s}$ ) at a height of 200 feet (about 66.6 meters) from the ground. The time and location mentioned in the report both match the airflow disturbance shown in the short-range LIDAR's scans. In addition, the short-range LIDAR data

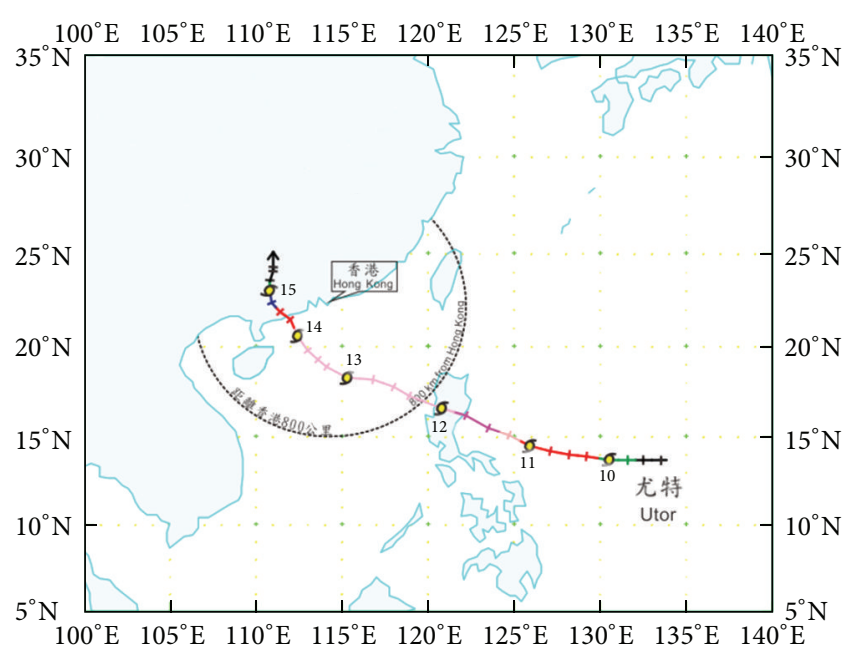

$\begin{array}{ll}\text { Hong Kong Observatory } & \\ - \text { Super typhoon } & - \text { Tropical storm } \\ - \text { Severe typhoon } & - \text { Tropical depression } \\ - \text { Typhoon } & -\sigma-\sigma-\text { Daily positions at 00 UTC }(08 \text { HKT) } \\ - \text { Severe tropical storm } & ++ \text { Intermediate 6-hourly positions }\end{array}$

FIGURE 2: Track map of tropical cyclone Utor (1311) on 9 to 16 August 2013.

shows that the airflow disturbance related to this windshear event was evolving so rapidly, with time scale of only a few dozen seconds, posing a challenge for the currently used long-range LIDAR scans of the airport over the take-off and landing glide paths, namely, with an average revisit time intervals of about 2 minutes only over a particular glide path.

\section{Performance of the Revised Alerting Algorithm}

Taking advantage of the high temporal and improved spatial resolution of the short range LIDAR employed in the 2013 field study, an improved alerting algorithm was devised, taking into account both spatial and temporal changes in the radial velocities measured along the arrival glide path. In the previous field study [2], automatic alerts were generated based on the magnitude of wind fluctuations (along 25RA) alone. During the 2013 campaign, it was observed that, in addition to large wind gradients, the movement of flow disturbances across the glide path also corresponded well with certain pilot windshear reports. Both effects were therefore considered in the revised algorithm such that an alert would be generated only when both spatial change and temporal change magnitudes exceeded their respective thresholds at a certain location simultaneously.

Denoting the measured radial velocity field by the SRL by $v(r, \theta)$, where $r$ and $\theta$ are the radial distance and azimuthal angle from the SRL, respectively, it is possible to extract the radial velocity profile $v_{\mathrm{GP}}(x)$, where $x$ is the position along the landing aircraft glide path, using a variety of numerical methods, for example, linear interpolation based on values from neighbouring grid-points. The spatial rate of change of radial 


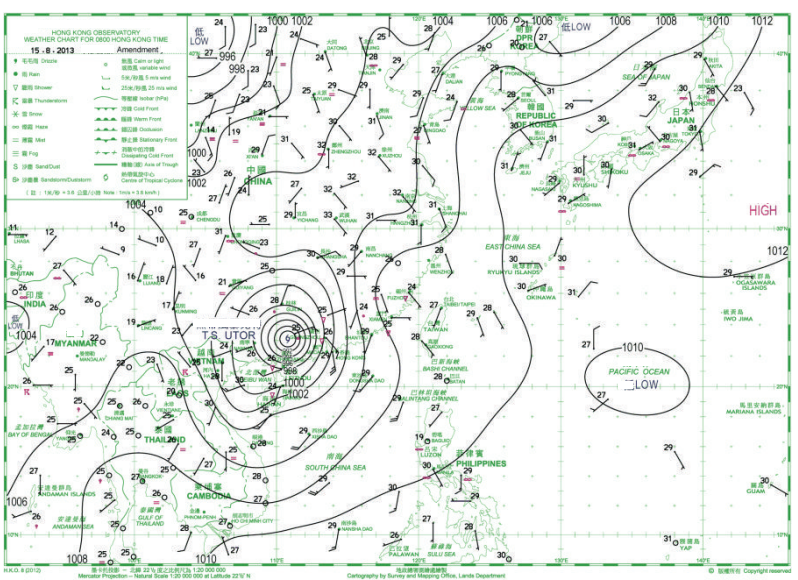

(a)

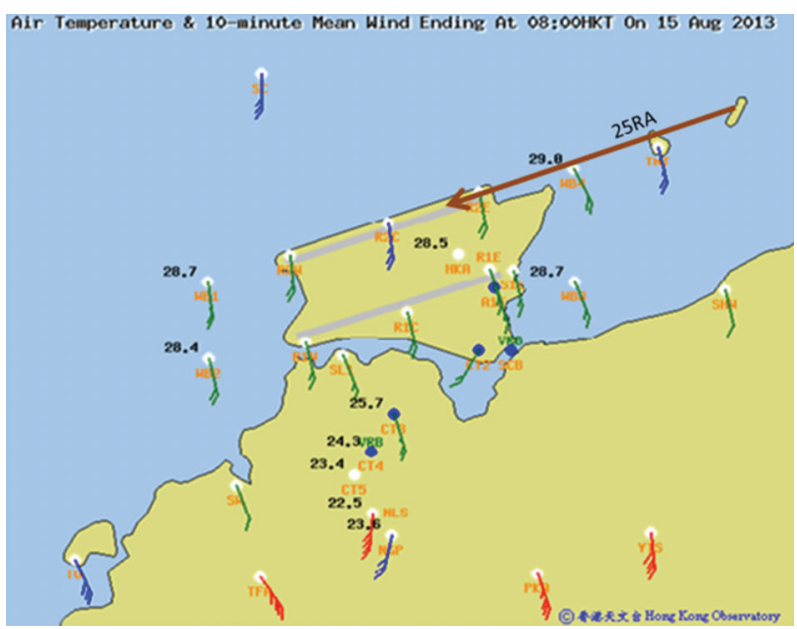

(b)

Figure 3: Weather overview at 8 am on 15 August 2013. (a) is a surface isobaric chart showing that tropical storm Utor is located at around $23.0 \mathrm{~N}, 110.8 \mathrm{E}$. (b) is an overview of wind speeds and directions as measured by the ground-based anemometers and weather buoys in the regions of Hong Kong International Airport and Lantau Island.

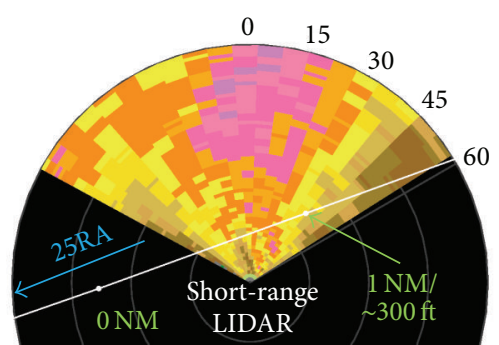

(a)

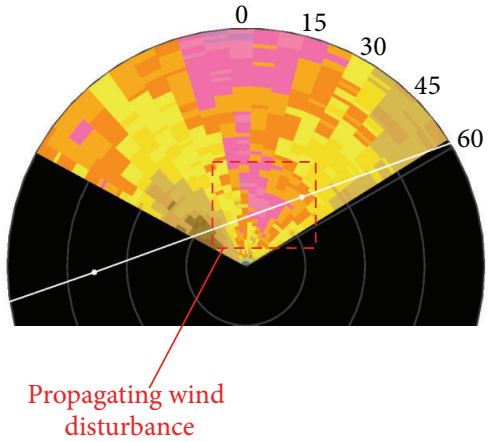

(b)

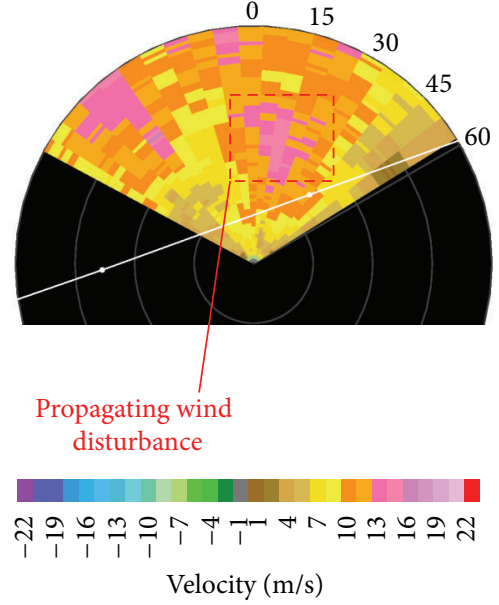

(c)

FIGURE 4: Radial velocity fields in the short-range LIDAR PPI. Each pixel in the image represents 30 meters in the radial direction, with time difference of 40 seconds between images. (a) shows a relatively even radial velocity field for background wind over the 25RA descent glide path. (b) shows airflow disturbance over the 25RA descent glide path. (c) shows airflow disturbance left from the 25RA descent glide path.

winds as would have been experienced by the pilot is then approximated by the spatial derivative of the velocity profile, that is, $\partial v_{\mathrm{GP}} / \partial x$. Note that, as a result of the scanning geometry of the SRL, the direction of the "radial" wind at each point along the glide path in fact differs slightly from its immediate neighbours and hence is not the true "crosswind" (wind component perpendicular to direction of aircraft motion) in the strictest sense. Similarly, the temporal rate of change in radial winds is estimated using the time derivative of the velocity profile. Assuming a time difference of $\Delta t$ between two consecutive PPI scans (and hence velocity profiles), the time derivate $\partial v_{\mathrm{GP}} / \partial t$ at each position $x$ can be approximated by $\Delta v_{\mathrm{GP}}(x) / \Delta t$, where $\Delta t$ is 20 seconds in the current study.
A threshold-based alerting algorithm is then applied simultaneously to the profiles of spatial and temporal changes such that an alert will be issued whenever the thresholds on both components of changes are exceeded. This can be expressed mathematically as

$$
\begin{aligned}
& \frac{\partial v_{\mathrm{GP}}}{\partial x} \geq T_{1}, \\
& \frac{\partial v_{\mathrm{GP}}}{\partial t} \geq T_{2},
\end{aligned}
$$

where $T_{1}$ and $T_{2}$ are alert thresholds (not necessary correlated) on spatial and temporal changes, respectively. Since each radial velocity profile covers a certain length in space, it 


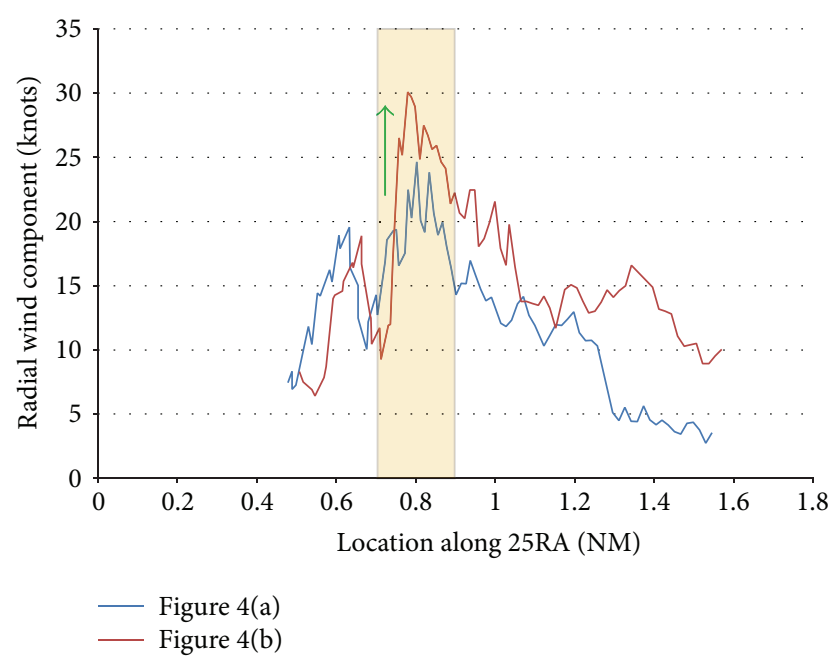

FIGURE 5: Profile of radial wind component measured by shortrange LIDAR along glide path of corridor 25RA during the windshear event shown in Figure 4. Blue line corresponds to the top panel of Figure 4 and red line the middle panel. The rapid surge in radial velocity associated with the pilot windshear report is highlighted.

is possible for $T_{1}$ and $T_{2}$ to be exceeded at different locations of the same profile (i.e., at the same time but not at the same location). For the purpose of the current study, an alert will be triggered as long as both $T_{1}$ and $T_{2}$ are exceeded at, at least one point of the same profile.

Using the airflow disturbance in Section 2 (Figure 4) as an example, the radial wind profiles along 25RA during the windshear event are shown in Figure 5. It could be seen that, during the passage of the disturbance, a sudden surge in wind speed occurred between 0.5 nautical mile (NM) and $1 \mathrm{NM}$ from the runway end, giving rise to sharp gradients in both the temporal and spatial components during that moment. Such features would be efficiently captured by the new alerting algorithm.

To investigate the performance of the revised algorithm, a relative operating characteristics (ROC) diagram will be employed (Figure 6). The vertical axis is the percentage of detection (POD), which is defined as (number of hits, i.e., windshear reports covered by short-range LIDAR alerts)/ (total number of events) $\times 100 \%$. The horizontal axis is the percentage of time on alert (POTA), which is defined as (amount of time with alerts in force)/(length of the study period) $100 \%$. The coloured dots represented the performance of the algorithm using different threshold sets. The dots were spread over an area since two parameters (thresholds for spatial and temporal changes) were in use. High skill level is indicated by positions close to the top-left corner, that is, high POD with relatively small number of alerts issued.

During the 2013 field study, a total of 23 windshear reports were received over corridor 25RA. Distribution of the coloured dots (along curves to the top-left corner) indicated that positive skill levels could generally be obtained from the new algorithm. For example, at an alert duration of about $10 \%$, a POD of $70 \%-75 \%$ could be attained depending on the thresholds. For comparison, the performance of the WTWS

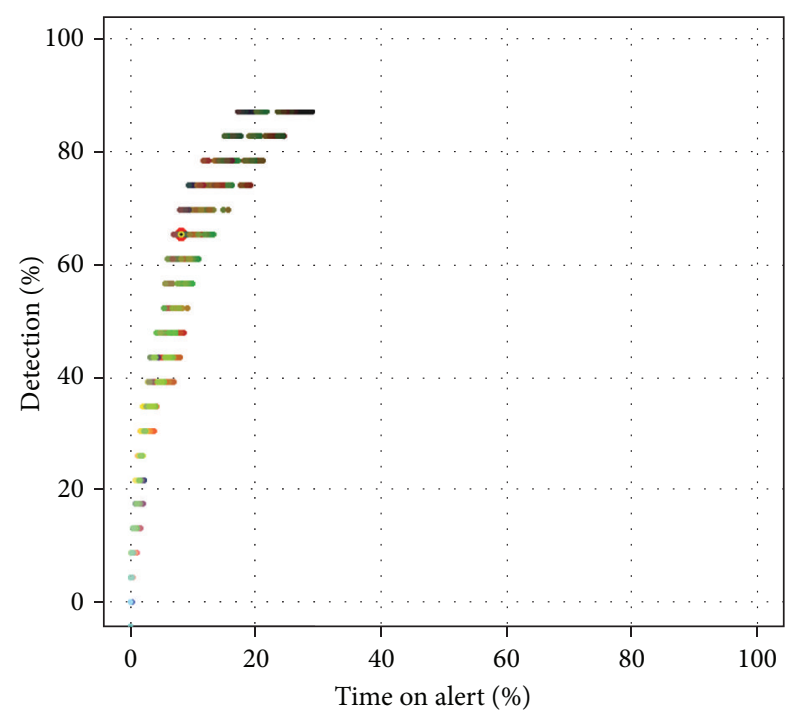

FIGURE 6: Early alerting ROC curve for windshear generated by a short-range LIDAR during the 2013 field experiment. The colored dots represent the performance of the new early alerting algorithm using a combination of thresholds for spatial and temporal changes in radial wind velocities. The red circle represents the performance of WTWS.

during the same period was represented by the Bull's Eye. Note that both long-range LIDAR systems at the Airport were under maintenance during most of the study period; hence the majority of WTWS alerts would have come from detection algorithms using anemometers near the Airport and on Lantau Island. Using suitable combination of alert thresholds, performance similar to or slightly better than WTWS (POD of $65 \%$ at POTA of $8 \%$ ) could be reproduced using the shortrange LIDAR. Depending on operational constraints (e.g., the relative importance or balance between maximizing hits and reducing false alarms), optimal threshold sets $\left(T_{1}, T_{2}\right)$ can be chosen by picking points on the ROC diagram with the desired level of performance. It could also be seen from the ROC diagram that the performance of the algorithm (i.e., location of each coloured dot) varies smoothly and in a continuous manner with respect to small changes in both thresholds, which suggests that the performance statistics are robust against fluctuations in the alert thresholds.

The results show that the revised alerting algorithm based on both spatial and temporal wind fluctuations along the glide path offers advantages in capturing low-level windshear events over corridor 25RA and potentially supplementing the operational WTWS at Hong Kong International Airport.

\section{Airflow Disturbance Evolution}

In order to better interpret the air disturbance, we use Lagrangian coherent structure (LCS) extraction algorithms to highlight the evolution of air disturbances seen in the shortrange LIDAR data. The building-induced flow structures, mainly in the form of coherent vortices, appear to pass through the LIDAR domain throughout the duration of 


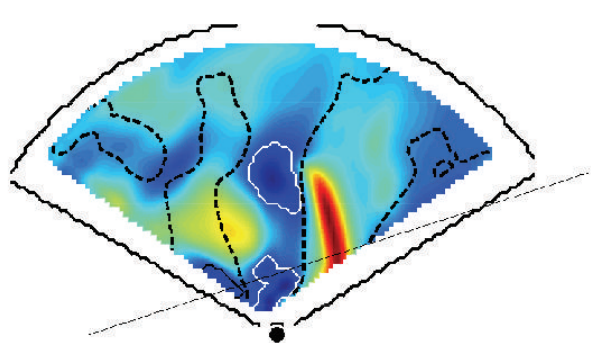

(a)

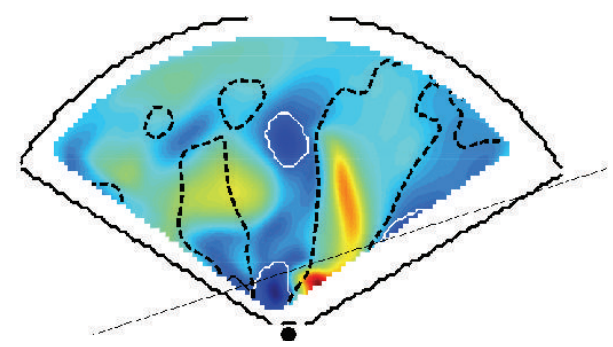

(b)

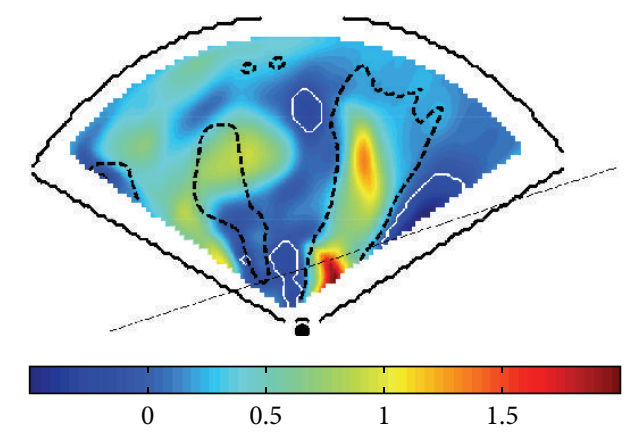

(c)

FIGURE 7: Evolution of an anticyclone depicted by the Lagrangian measure, around 1:50 pm on 15 August (Hong Kong time). (a)-(c) are shown in consecutive order by 20 -second intervals. The color plots are the Lagrangian diagnostic, which show coherent vortices when the diagnostic is blow 0 (deformation tensor exhibits complex eigenvalues). The black contours show actual LIDAR coverage. The dashed black contours separate regions of different rotation direction, irrespective of whether vorticity dominates strain. The white isocontours enclose regions with anticyclones; that is, these regions are vorticity dominant and rotate in the clockwise direction. The black straight lines indicate 25RA. In this event, anticyclones pinch off from around the LIDAR and move northwards, whereas new anticyclone patches pinch off across the runway.

the field study period. A Lagrangian detection method is preferred as it measures regions of the domain that persistently exhibit coherent fluid motion, as compared to instantaneous Eulerian based methods, and distinguishes airflow disturbances that are robust against small errors in space and time [3]. Previously, a Lagrangian method based on the finitetime Lyapunov exponents has been developed to extract structures associated with high stretching and shear regions [4]. In this study, we adopt a new method suitable for the data set that distinguishes rotation regions over 1-2 minutes.

The method is the compressible version of a recent mixing diagnostic, the mesoellipticity measure, developed to study the 2010 Gulf of Mexico Oil Spill [5]. The mesoellipticity method examines the gradient tensor of the Lagrangianly averaged velocity along fluid parcel trajectories. If the trace and determinant of the velocity gradient tensor $\operatorname{Tr}$ and $\Delta$ satisfy that $\operatorname{Tr}^{2}-4 \Delta<0$, then the tensor exhibits complex eigenvalues, indicating persistent rotation over the time of integration. In [5], the authors further restricted the analysis to incompressible flows, where $\operatorname{Tr}$ and $\Delta$ can be directly related and the diagnostic further simplified. The restriction of incompressibility is lifted here to allow the examination of two-dimensional PPI scans of the three-dimensional domain, which is inherently compressible. In this algorithm, a twodimensional variational retrieval method is applied to the line-of-sight (LOS), or radial, data to obtain the corresponding two-dimensional velocity field. Virtual fluid parcel trajectories are then integrated over time to obtain the deformation tensor along the PPI scan (cf. details in [4]). A transition zone is used to smoothly connect the observed data from inside and the extrapolated data from outside of the LIDAR domain. As such, the Lagrangian analyses area is smaller compared to actual LIDAR coverage. In this methodology, a rotating region is identified as where the gradient tensor exhibits complex eigenvalues. Operationally, this leads to the diagnostic of mesoellipticity whereever $M=\operatorname{Tr}^{2}-4 \Delta<0$. The integration time of trajectories is chosen to be 2 minutes. This roughly corresponds to the time where the virtual fluid trajectories travel through the short-range LIDAR domain.

Note that a more rigorous, material-based method for vortex extraction has been developed very recently [6]. It is based on the requirement that trajectories form closed orbits over long enough integration time, so material transport is prohibited from inside and outside of the orbit. Our method is appropriate for the case of interest because, for the duration that trajectories travel through the small domain, there is no chance for such closed orbits to form. It still supersedes instantaneous measures, such as the Okubo-Weiss parameter, as it incorporates 6 frames of flow data.

We highlight a case around 1:50 pm on 15 August (Hong Kong time) in Figure 7, where a series of anticyclone patches crosses the northern runway. The color plot in each panel shows the mesoellipticity measure, which is essentially a test whether strain dominates vorticity or vice versa along the examined trajectory. When it falls below 0 , the gradient tensor exhibits complex eigenvalues and the flow is coherently 
rotating. In addition to the mixing diagnostic, we also obtain the angle of rotation to determine if the rotation is cyclonic or anticyclonic. The black dashed contour separates the domain into cyclonic and anticyclonic regions, irrespective of whether vorticity dominates strain. Under this partition, we use thin white isocontours to indicate anticyclones and thin black isocontours to indicate cyclones (not seen at the time shown due to lack of cyclones). The thick black isocontours indicate the edge of LIDAR coverage. The grid resolution is chosen to be 30 meters in both horizontal directions, matching the finest LIDAR resolution. The structures are robust and subject to quadruple resolution in both horizontal directions.

The evolution of one patch is shown as a sequence from Figures $7(\mathrm{a})-7(\mathrm{c})$, with 20 -second intervals between each plot. The patch is enclosed in the white isocontour, indicating that the rotating region is an anticyclone. This patch is originally connected to the anticyclone patch near the LIDAR center. In Figure 7(a), it is seen just north of 25RA (black straight line), whereas it moves northward at later times. In general, these patches are on the order of a few hundred meters and have a lifetime of a few minutes. These scales are hard to resolve when only long-range LIDAR systems are considered. Note also that even though cyclones are not seen in this figure, they are present at other times of the field study.

To understand the passing rotating structures better, we plot a Hovmoller diagram to show the time history of structures passing the landing trajectory. The measure we use is derived from the mixing diagnostic. We first filter out the regions whose diagnostic values are larger than 0 . For those regions less than 0 , we set the diagnostic values to reverse sign if the rotation measure shows cyclone. In this way, the Hovmoller diagram only shows rotation features, with negative values indicating anticyclones and positive values indicating cyclones.

The Hovmoller diagram is generated between $1 \mathrm{pm}$ and 2:40 pm on 15 August (Hong Kong time), shown in Figure 8. It is very interesting to note that anticyclones are predominant during this time period, with the presence of a couple of weaker cyclones. At the end, a few stronger cyclones also appear. Along the landing trajectory, they are observed to propagate away from the runway threshold, although it is clear that the actual structure evolution is more from south to north as seen in Figure 7. At any given time, the structures (as seen along the landing trajectory) are about 0.1-0.2 nautical miles or about 200-300 meters. The time for them to cross the runway is only a minute or two. This corroborates the importance of short-range LIDAR in detection of these small-scale features.

Another interesting feature of this diagram is the relatively well-defined periodicity in the occurrence of rotating structures. A spectral analysis near the bottom of Figure 8 (0.5 NM from runway threshold) shows a relatively dominant period of about 5 minutes during this time, although, for major features with dark blue stripes, a period of about 20 minutes is seen in the spectral peaks. This periodicity may indicate the origin of these eddies.

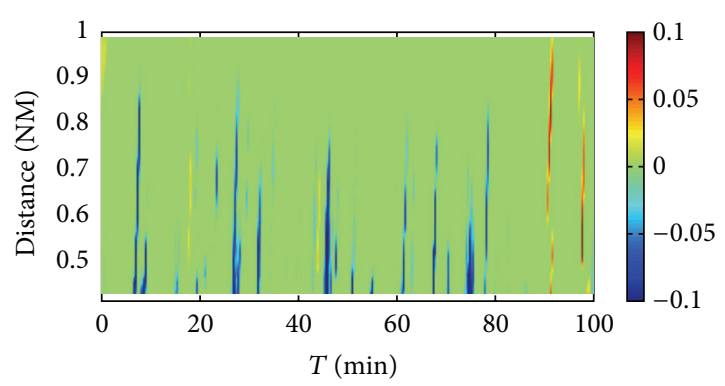

Figure 8: Hovmoller diagram of the filtered diagnostic field along the runway 25RA. The distance is from runway threshold in nautical miles.

\section{Summary}

This paper analyzed the application of a short-range LIDAR in detecting windshear event over Hong Kong International Airport Runway 25RA during the field experiment held in the summer of 2013. Since the short-range LIDAR has relatively high spatial and temporal resolution, it can detect brief and rapidly changing airflow disturbances to effectively capture low altitude windshear and turbulence brought about by buildings. During the field experiment in the summer of 2013, the Observatory used the improved short-range LIDAR windshear early alerting algorithm, plus controlling different radial wind speed spatial and temporal change thresholds. The derived ROC curve shows that the new early alerting methodology offers some advantages in capturing low altitude windshear reports over runway 25RA. The newly developed Lagrangian algorithm captures the essential coherent motion of air disturbances. Hovmoller diagram of the processed mixing diagnostic reveals further characteristics of the eddies passing the landing trajectory. The Observatory will continue to conduct field experiment in 2014 to improve and verify the short-range LIDAR windshear early alerting algorithm, striving to make it more accurate and more effective at giving early alerting for windshear.

\section{Conflict of Interests}

The authors declare that there is no conflict of interests regarding the publication of this paper.

\section{Acknowledgment}

Wenbo Tang acknowledges the support from NSF Grant ATM-0934592.

\section{References}

[1] C. M. Shun and P. W. Chan, "Applications of an infrared Doppler lidar in detection of wind shear," Journal of Atmospheric and Oceanic Technology, vol. 25, no. 5, pp. 637-655, 2008.

[2] P. W. Chan and Y. F. Lee, "Application of short-range lidar in wind shear alerting," Journal of Atmospheric and Oceanic Technology, vol. 29, no. 2, pp. 207-220, 2012. 
[3] G. Haller, "Lagrangian coherent structures from approximate velocity data," Physics of Fluids, vol. 14, no. 6, pp. 1851-1861, 2002.

[4] W. Tang, P. W. Chan, and G. Haller, "Lagrangian coherent structure analysis of terminal winds detected by lidar. Part I: turbulence structures," Journal of Applied Meteorology and Climatology, vol. 50, no. 2, pp. 325-338, 2011.

[5] I. Mezic, S. Loire, V. A. Fonoberov, and P. Hogan, "A new mixing diagnostic and gulf oil spill movement," Science, vol. 330, no. 6003, pp. 486-489, 2010.

[6] G. Haller and F. J. Beron-Vera, "Geodesic theory of transport barriers in two-dimensional flows," Physica D: Nonlinear Phenomena, vol. 241, no. 20, pp. 1680-1702, 2012. 

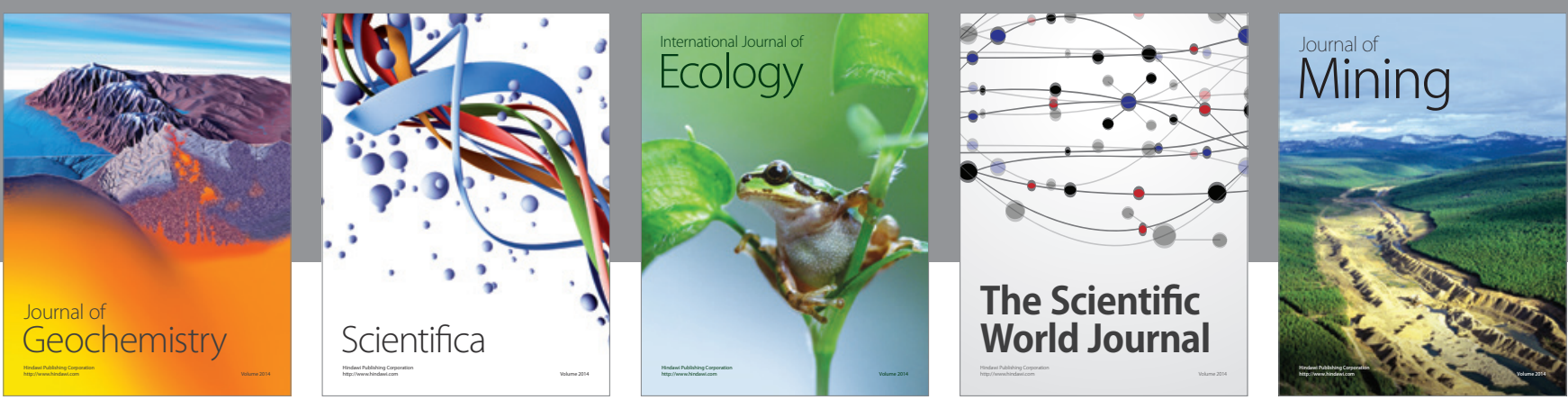

The Scientific World Journal
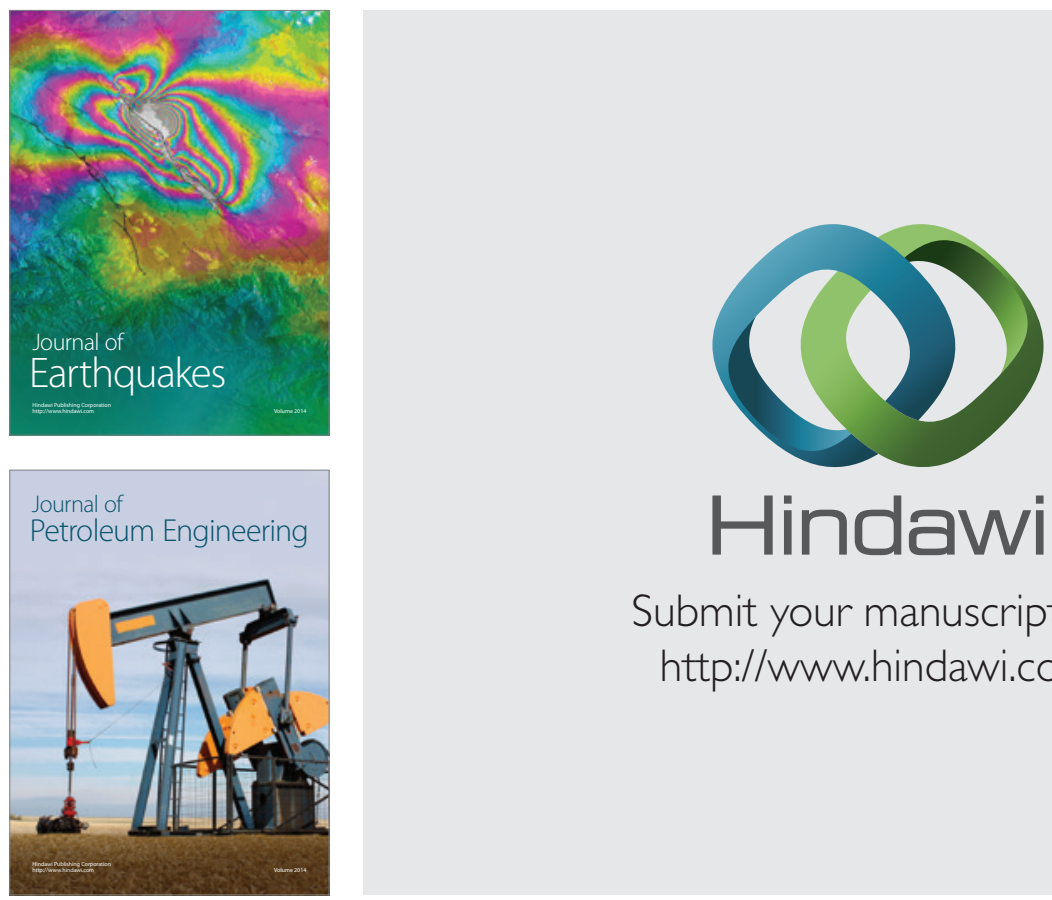

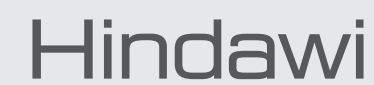

Submit your manuscripts at

http://www.hindawi.com
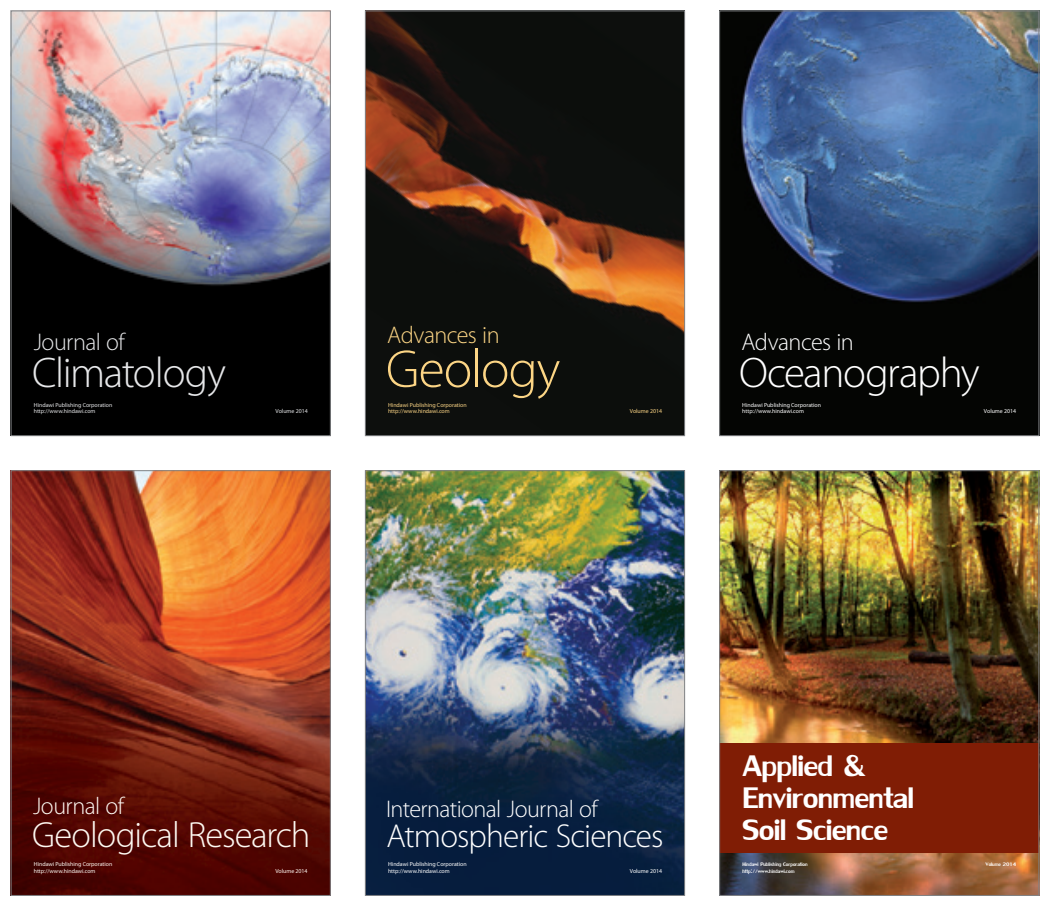
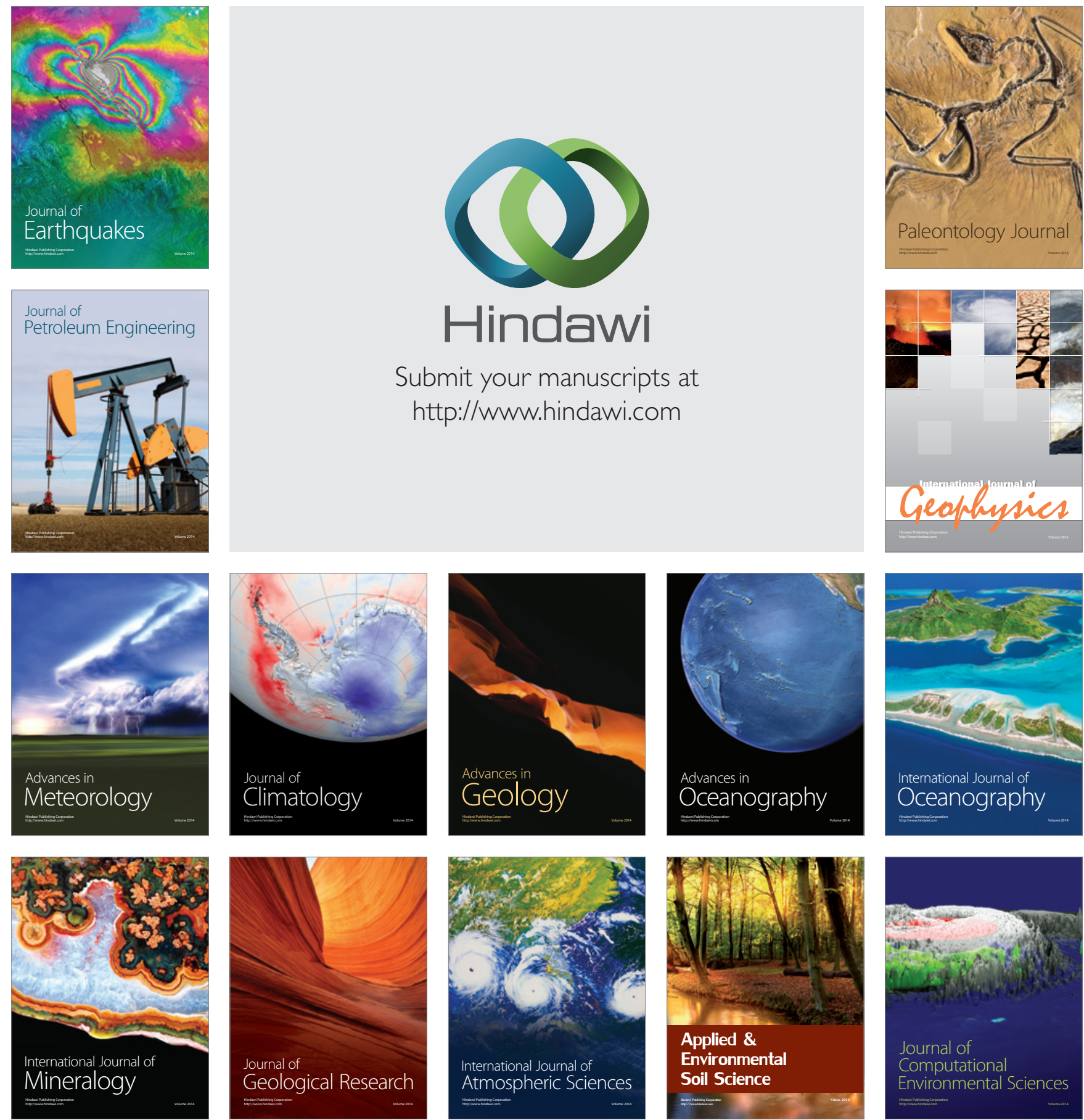